\section{Metabolic and nutritional approach to older frail people}

\author{
Stefano Volpato, Giovanni Zuliani \\ Department of Medical Science, \\ University of Ferrara, Italy
}

\begin{abstract}
Frailty is a common clinical syndrome in older adults that carries an increased risk for poor health outcomes including falls, incident disability, hospitalization, and mortality. It is characterized by multisystem dysregulations, leading to a loss of dynamic homeostasis, decreased physiologic reserve, and increased vulnerability to stressors. A large body of literature suggests several important multisystem pathophysiologic processes in the pathogenesis of the frailty syndrome, including chronic inflammation and immune activation, insulin resistance and those in musculoskeletal and endocrine systems. Currently, no effective pharmaceutical interventions have been developed for the prevention and treatment of the frailty syndrome. Conversely, epidemiological and intervention studies suggest that adequate nutrition and physical exercise might prevent or postpone the onset of frailty and related clinical manifestations.
\end{abstract}

\section{Metabolic and nutritional approach to older frail people}

Frailty has been defined as a geriatric syndrome, indicating the accumulated effect of impairments in multiple domains that all together result in a particular adverse outcome. ${ }^{1}$ Frail individuals have a higher risk of developing disabilities in basic and instrumental activities of daily living, falling, institutionalization, and death. Although different definitions have been proposed, two themes seem to better depict the frailty concept: i) loss of functional reserve in multiple areas/domains; ii) existence at a level close to or past the threshold for failure, with reduced tolerance to common stressors.

Most definitions of frailty involve decline in mobility, strength, endurance, nutrition, and physical activity as clinical components, while others include cognitive impairment and depression too. ${ }^{2}$ Thus, there are several domains involved in frailty development including neurological control/cognition, mechanical performance/mobility (muscle/bone strength, joint function, balance, coordination, and motor processing), energy metabolism (nutrition and cardiopulmonary function), and physical activity. Frailty might be viewed as the result of acceleration in the progressive decline of multi-systemic homeostatic reserve related to aging, genetic, epigenetic, and environmental factors. A current pathogenetic hypothesis of frailty is based on interaction between aging and multiple chronic diseases. Aging itself is characterized by reduced reserve across different homeostatic systems; as a result, a multi-systemic impairment related to the accumulation of molecular and cellular damage, and loss of feed forward and feedback mechanisms among interacting systems is observed. ${ }^{3}$ Multiple chronic diseases superimpose the weakened homeostatic systems by further reducing cross-system compensation, and favoring the onset of frailty.

Starting from current pathogenetic hypotheses, the prevention and treatment of frailty should be aimed at contrasting the conditions consistently associated with frailty development, including the multisystemic impairment related to aging itself and the multi-systemic effects of multimorbidity and sub-clinical diseases. ${ }^{4}$

The key points of population-based approach to frailty would be the identification of a pathogenetic background common to frailty phenotype and related chronic diseases. In this regard, Walston et al. ${ }^{5}$ proposed a biological model in which different molecular mechanisms together with agingrelated chronic diseases would lead to a condition of systemic inflammation, insulin resistance, and oxidative stress, which in turn would result in the development of frailty $^{6}$ (Figure 1). Genetics might influence this process; indeed, an association between frailty and several mitochondrial DNA variations has been reported. Nevertheless, lifestyle seems to have a central role; it has been suggested that smoking, elevated BMI, and poor physical exercise during adulthood predict disability and earlier mortality (two outcomes of frailty) in older age; furthermore, commonly used CVD risk scores in the adulthood similarly predict the risk of frailty.

Currently, no effective pharmaceutical interventions have been developed for the prevention and treatment of the frailty syndrome. Conversely, epidemiological and intervention studies suggest that adequate nutrition and physical exercise might prevent or postpone the onset of frailty and related clinical manifestations. ${ }^{7}$ There is strong evidence that over-nutrition in adulthood leads to systemic inflammation, insulin resistance, and oxidative stress, and
Correspondence: Stefano Volpato,

Department of Medical Science, University of Ferrara, via Ariosto 35, I-44121 Ferrara, Italy. Tel.: +39.0532.236658;

Fax: +39.0532 .210884$

E-mail: vlt@unife.it

Key words: Frailty; nutrition; prevention; epidemiology.

Conference presentation: paper presented at $31^{\circ}$ Congresso Nazionale SIGOT - Genova, June 8-9, 2017

Received for publication: 8 September 2017 Accepted for publication: 15 September 2017.

This work is licensed under a Creative Commons Attribution-NonCommercial 4.0 International License (CC BY-NC 4.0).

CCopyright S. Volpato and G. Zuliani, 2017 Licensee PAGEPress, Italy

Geriatric Care 2017; 3:7056

doi: $10.4081 /$ gc. 2017.7056

finally to increased risk of developing frailty; conversely, the adherence to a Mediterranean diet has been associated with lower systemic inflammation, insulin resistance, and oxidative stress, in turn reducing the risk of frailty. ${ }^{8}$ It has to be underlined, however, that in older age the deficiency of nutrients, and not their excess, might increase the risk of becoming frail; indeed, the lack of fundamental components, such as essential amino acids, has been implicated in the development of sarcopenia, while macro-nutrient supplements have been shown to be helpful in maintaining muscular protein metabolism and cellular function. It is not just the lack of proteins, but also a general lack of nutrients that might lead to an increased risk of frailty in older age. Frailty has been associated with low levels of micronutrients, including vitamin $\mathrm{E}$ and vitamin D. Dairy products (i.e., milk, cheese, yoghurt) might be also important for maintaining physical performance and avoiding sarcopenia; a recent study found that older women eating more dairy products had significantly greater whole body lean mass, appendicular skeletal muscle mass, and greater handgrip strength. ${ }^{9}$

Apart from the dietary intake, several macro/micronutrient supplementations have been considered to exert a possible role in the prevention of frailty. With regards to primary prevention, a six-month DHA and EPA supplementation trial induced an improvement in walking speed compared to placebo, explaining more than $13 \%$ of the variance in the change in walking speed. On the other hand, several authors demonstrated the 


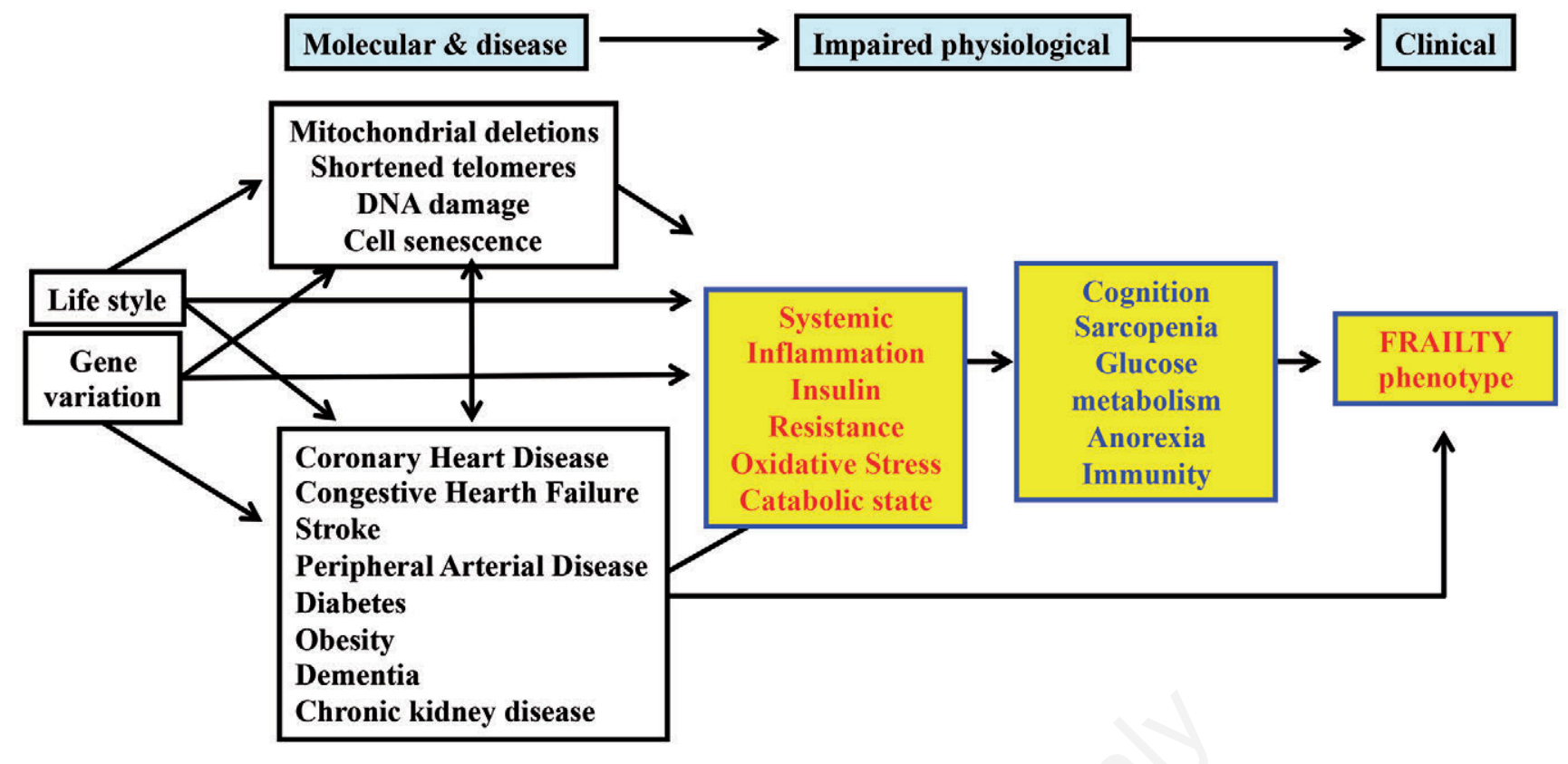

Figure 1. Schematic representation of the pathophysiology of frailty.

effectiveness of Vitamin D supplementation in maintaining physical performance, suggesting its therapeutic role in improving neuromuscular function and reducing not only fractures after falls, but also the incidence of falls. Protein supplementation is an important preventive-therapeutic intervention against functional decline, especially in frail elderly with malnutrition. It should be associated with physical exercise (both endurance- and resistance-type exercises). Protein supplementation should take place just after physical exercise, to take advantage of its sensitizing effect, and should contain at least 2-2.5 g of leucine.

Decline in physical activity with aging is associated with a decrease in exercise capacity that predisposes to frailty. The frailty syndrome includes a lowered activity level, poor exercise tolerance, and loss of lean body and muscle mass. There are multiple mechanisms by which regular physical activity protects against chronic inflammation-related diseases: regular exercise induces the reduction of visceral fat mass, which is known to be associated with inflammation and insulin resistance; furthermore, at the molecular level, exercise might reduce frailty by decreasing muscle inflammation, increasing anabolism, and increasing muscle protein synthesis.

Aerobic endurance training can significantly improve peak oxygen consumption by $\sim 10-15 \%$. Resistance training is the best way to increase muscle strength and mass. Although the increase in muscle mass in response to resistance training may be attenuated in frail older adults, resistance training can significantly improve muscle strength, particularly in institutionalized patients, by $\sim 110 \% .{ }^{10}$ Because both aerobic and resistance training target specific components of frailty, studies combining aerobic and resistance training provide the most promising evidence with respect to successfully treating frailty. Based on the available studies, an individualized multicomponent exercise program that includes aerobic activity, strength exercises, and flexibility is recommended to treat frailty. More studies, however, are needed to determine which exercises are best suited, most effective, and safe for this population.

\section{References}

1. Morley JE, Haren MT, Rolland Y, Jong Kim M. Frailty. Med Clin N Am 2006;90:837-47.

2. Fried LP, Tangen CM, Watson J, et al. Frailty in older adults: evidence for a phenotype. J Gerontol 2001;56:M146-56.

3. Clegg A, Young J, Iliffe S, et al. Frailty in elderly people. Lancet 2013;381:752-62.

4. Zuliani G, Soavi C, Maggio M, et al. Counteracting inflammation and insulin resistance with diet and exercise: a strategy for frailty prevention? Eur Geriatr Med 2015;6:220-31.

5. Waltson J, Hadley EC, Ferrucci L, et al. Research agenda for frailty in older adults: toward a better understanding of physiology and etiology: summary from the American Geriatrics Society/ National Institute on Aging Research Conference on frailty in older adults. J Am Geriatr Soc 2006;54:991-1001.

6. Barzilay JI, Blaum C, Moore T, et al. IR and inflammation as precursors of frailty: the Cardiovascular Health Study. Arch Intern Med 2007;167:635-41.

7. Michel JP, Cruz-Jentoft AJ, Cederholm T. Frailty, exercise and nutrition. Clin Geriatr Med 2015;31:375-87.

8. Talegawkar SA, Bandinelli S, BandeenRoche K, et al. A higher adherence to a Mediterranean-style diet is inversely associated with the development of frailty in community-dwelling elderly men and women. J Nutr 2012;142:2161-6.

9. Radavelli-Bagatini S, Zhu K, Lewis JR, et al. Association of dairy intake with body composition and physical function in older community-dwelling women. J Acad Nutr Diet 2013;113:1669-74.

10. Fiatarone MA, O’Neill EF, Ryan ND, et al. Exercise training and nutritional supplementation for physical frailty in very elderly people. N Engl J Med 1994;330: 1769-75. 